\title{
Continuous Health Monitoring Using E-Textile Integrated Biosensors
}

\author{
Catarino, André, Carvalho, Helder, Dias, Maria J., \\ Pereira, Tiago \\ Department of Textile Engineering \\ University of Minho \\ Guimarães, Portugal \\ helder@det.uminho.pt
}

\author{
*Postolache, Octavian, ${ }^{* *}$ Girão Pedro S. \\ Instituto de Telecomunicações /*ISCTE-IUL, ${ }^{* *}$ DEEC-IST \\ Lisbon, Portugal \\ opostolache@1x.it.pt, psgirao@ist.utl.pt
}

\begin{abstract}
In this work, continuous health monitoring for disabled or elderly people is proposed using textile integrated electrodes for ECG measurement. Other applications, such as EMG or skin impedance measurement are also envisaged. The paper specifically describes a shirt integrating electrodes for ECG measurement that has been tested in several conditions. The techniques for integration of ECG electrodes can be directly applied for production of EMG or skin-impedance electrodes. Signal processing techniques for heart rate value extraction and to deal with low-quality signal or motion artefacts are being tested and will also be described. Results show that signals acquired with the shirt are comparable to signals acquired with conventional gel electrodes. The complete integration of the electrodes into clothing may have a very interesting psychological benefit, but some issues related to comfort and daily use have to be further investigated.
\end{abstract}

\section{Keywords - electrocardiography, e-textiles, health monitoring}

\section{INTRODUCTION}

At present time, considering the ageing of the modern societies' populations, the interest for ubiquitous, continuous and automatic health monitoring has been growing intensely. One of the technologies that have captured some attention in this context is the integration of sensors and systems into textiles. Textiles are excellent interfaces for biological signal sensing. They are flexible, conformable and are used by everyone on a daily basis. There are many types of textile conductive yarns available on the market that can be processed by conventional textile production techniques. Many researchers have studied the realisation of sensors for health monitoring using textile materials and technologies, such as extension sensors for monitoring breathing rate and movement [1][2][3], electrodes for physiological signal sensing, such as ECG, EMG or skin impedance [3][4][5][6][7], moisture sensing [8]. In these studies some limitations have been observed [2][8], but the performance of textile electrodes as biosignal interfaces has generally been found - at least satisfactory [4][6][7].

In this work, a solution for ECG monitoring based on weft knitting technology and several elements regarding the conditioning circuit design for a future implementation of mobile ECG monitor are presented.

Acknowledgements: The authors wish to thank FCT, which funded work in this research through project Bioswim PTDC/EEA-ELC/70803/2006, pluriannual funding programmes and Instituto de Telecomunicações, Polo de Lisboa

\section{PRODUCTION OF THE SHIRT}

A MERZ model MBS seamless knitting machine was used equipped with a single needles system, located in the cylinder. The disk is equipped with transferring jacks, knives and springs which allow the control of the yarn in terms of going in or out the knitting zone. This machine is also a jacquard machine, with which it is possible to obtain complex structures with localised variations. This feature has been used to produce a particularly voluminous structure at the area used as electrode. In this way, the electrode area protrudes from the rest of the fabric, improving contact between the skin and the electrode, which is known to be a problem when using compression garments to stabilize the electrode's position.

The electrode areas are knitted with Elitex, which is a textured multifilament polyamide yarn with a thin silver coating (less than $10 \mathrm{~nm}$ ). They present very low values of electrical resistance (in the order $30 \Omega / \mathrm{m}$ ).

The same knitting technology allows the integration of electrical connections in the textile substrate. Due to the process that involves the insertion of the yarn into the knitting zone and the individual movement of the knitting elements, namely the needles, horizontal connections are easier to produce. Vertical connections are possible, but due to the specificities of this particular machine, imply a much higher consumption of the expensive conductive yarn used. Instead, conduits are created with the non-conductive yarns, through which conventional electrical conductors can be brought in.

Electrical insulation, if necessary, is achieved by coating with specific silicone compounds (Elastosil® from Wacker). This specific silicone presents a high adhesion capability, high electric isolation and stretch when compared with others.

Fig. 1 and 2 show pictures of the shirt. The shirt has three electrodes, the upper and middle electrodes being the sensing electrodes and the lower being the right-leg drive electrode. The placement of the electrodes has been chosen to avoid muscles, thus avoiding EMG signal interference. For better signal quality, the shirt has been constructed so that it has a tight fit. In order to obtain a good contact and stabilization of the electrode, the shirt was produced with polyamide and bare elastane. These two raw materials combined with the knitted structure allowed localised compression effects and thus reduce 
some artefacts that are present with body movement. On the other hand, the use of the Elitex yarn is due to its stable behaviour in terms of impedance even when subjected to stress. Since the electrodes are built into the fabric, when dressed, the electrodes stretch, which usually result in a modification of the electrode's impedance. It was observed that for the structures studied there was no evidence of a modification in the electrode's impedance when stretched, if the raw material was Elitex.

The electrical connections have, in a first phase, not been joined with the electrodes, to enable the study of signal quality before and after the textile conductor. To enable connections, standard snap fasteners have been applied. In a later stage, one of the possibilities would be to route the ECG connections to the back of the shirt and from there down to a single place to connect to the specific conditioning circuits, acquisition, primary processing and wireless communication module. To estimate the performance of the implemented T-shirt an ECG integrated conditioning circuit from Texas Instruments and an ECG measurement equipment, P-OX100 from Medlab, were used. Elements of the implemented conditioning circuit are following presented.

\section{SIGNAL CONDITIONING AND ACQUISITION}

An ECG measures the voltage generated by the contraction of the heart muscles. The signal conditioning challenges inherent to this application are primarily due to the small signal amplitude of only $0.2 \mathrm{mV}$ to $2 \mathrm{mV}$ peak-to-peak, the $0.05 \mathrm{~Hz}$ to $150 \mathrm{~Hz}$ bandwidth, and the $50 \mathrm{~Hz} / 60 \mathrm{~Hz}$ interference. In the present case an integrated solution based on ADS 1198 from Texas Instruments was considered.

The ADS 1198 integrated circuit from Texas instruments is characterized by eight low noise PGAs (programmable gain up to 12 ) and eight 16 bit resolution delta-sigma analog-to-digital converters. The integrated circuit includes a SPI compatible serial interface for communication interfacing. Taking into account the low level of amplification, a preamplifier is included in the system assuring a global gain of 336 combining a $G_{\text {pream }}=28$ of the preamplifier with $G_{A D S}=12$ of the ADS 1198 PGA.

The preamplifier is a differential ac-coupled amplifier implementing a differential bandpass filter, based on the work by Spinelli et al [9]. It assures high input impedance with very low CMRR. The bandwidth has been chosen for a preliminary study of the system's behaviour regarding motion artefacts and sensitivity to EMG interference, but in a final version it should be reduced to a low-pass cut-off at the usual $100-150 \mathrm{~Hz}$ for ECG acquisition.

The ECG testing system set-up was done using a SPI-USB interface (NI USB-8451) that permits the communication between a PC and the ADS 1198. Through the specific commands transmitted through the SPI bus, the acquisition rate and the gain associated with ADS 1198 control are imposed. This control corresponds to ADS-SPI software module developed in LabVIEW that it is a part of an ECG acquisition and processing software implemented at a PC level.

Referring to the reference ECG measurement system that was used to highlight the capabilities of E-textile dry electrodes, a digital pulse oximeter with ECG (P-OX100) was used. A set of analog signals corresponding to the digital-toanalog converter module attached to the P-OX100 are acquired using an NI USB-6008 that permits the signal processing and data storage of the ECG signals.

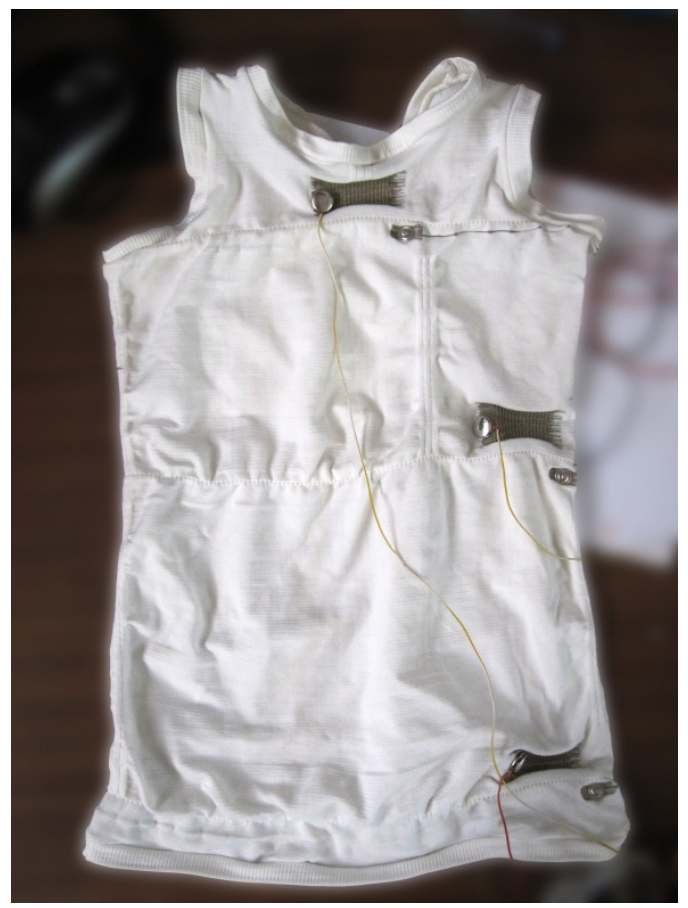

Figure 1. Shirt with electrodes connected directly to cables.

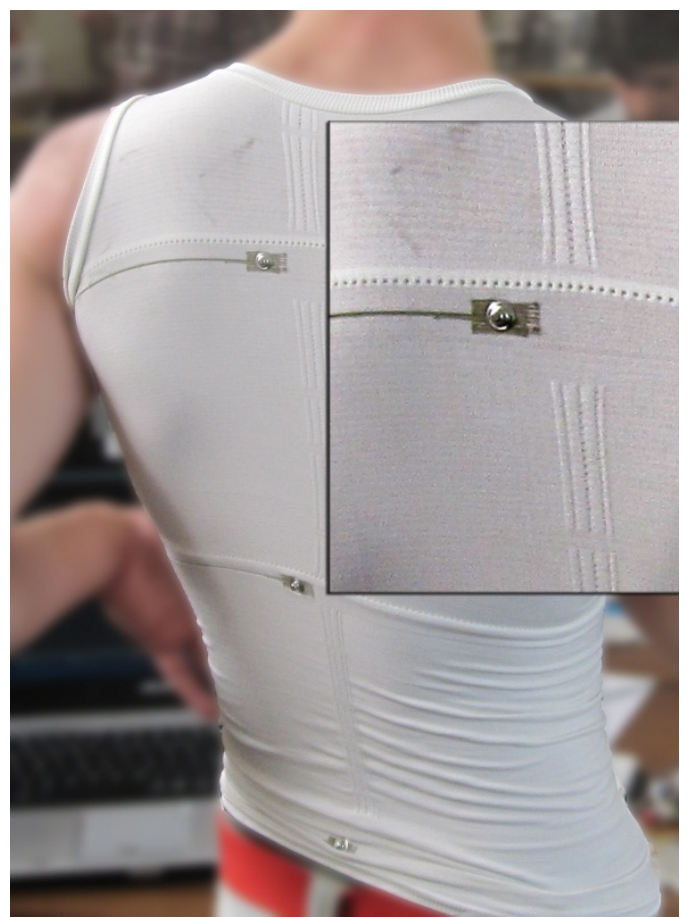

Figure 2. Detail of horizontal connecting lines and vertical cable conduits.

The P-OX100 reference equipment includes amplification, analog filtering and digital filtering capabilities providing also information such as heart rate and $\mathrm{SpO} 2$ that are available in 
digital but also in analogue form to be acquired using USB6008 and displayed by the software GUI.

The ADS 1198 is then connected to a NI-8451 board for SPI interfacing with LabVIEW, on which an application for data collection has been developed. The system is represented in Fig. 3.

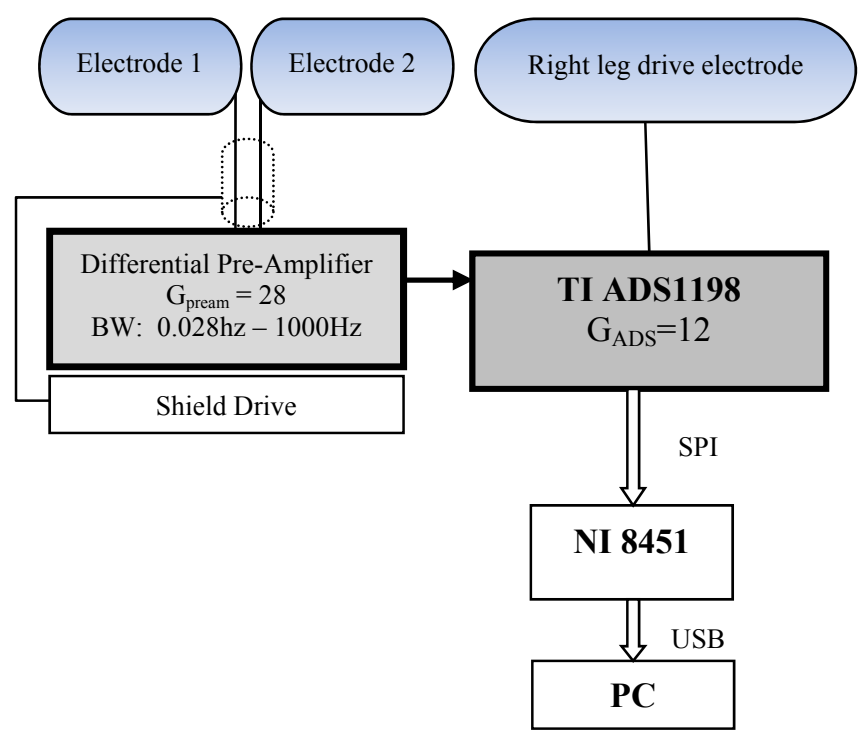

Figure 3. Block diagram of e-textile ECG electrode based on TI ADS1198 integrated solution.

\section{SIGNALS}

Figs. 4 and 5 show two representative examples of signals acquired using the E-textile electrodes embedded on the T-shirt and using the ECG acquisition system based on ADS1198. In the first case, the shirt and the electrodes are completely dry, whilst the electrodes were then wetted with plain water.

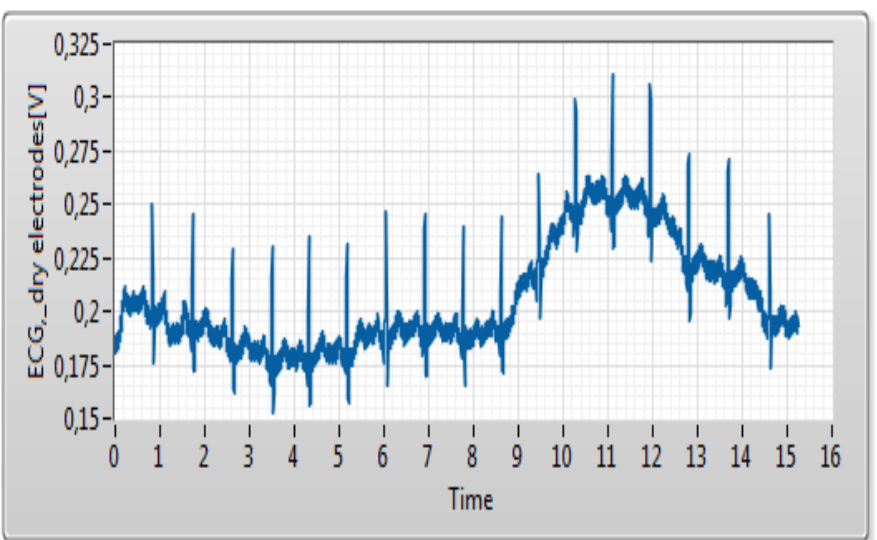

Figure 4. Signal acquired with dry electrodes.

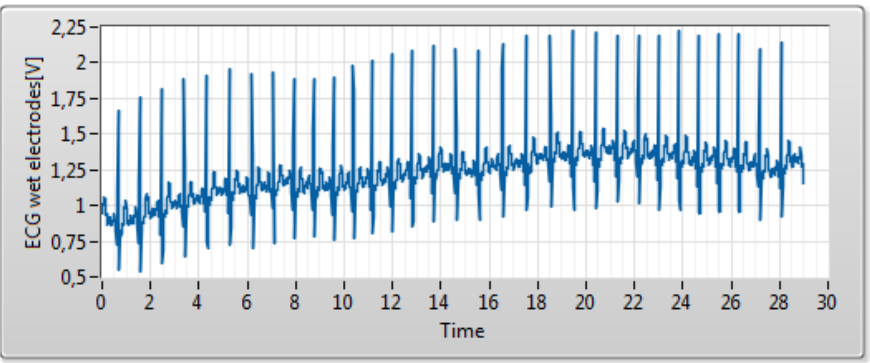

Figure 5. Signal acquired with wet electrodes.

As can be clearly observed, there is a tenfold increase of signal amplitude when the electrodes are wetted. There are artefacts caused by subject's motion but also by breathing activity. Using the wet electrodes the ECG signal becomes much more stable and much less noisy. However, this may increase the discomfort and can cause important wave amplitude change in long term monitoring. It has been observed that despite the tight fit of the shirt the upper electrode still does not adequately compressed against the body. To acquire the signal presented in Fig. 4 the user had to compress the electrode slightly. This is an aspect that has to be carefully reviewed and analysed in function of the end-user.

Additional tests were done using the T-shirt with dry electrodes connected to the Medlab P-OX100 reference system. The signals were acquired for a standard period of 5 minutes, the P-OX100 output signal corresponding to the 3 lead ECG being acquired at a 500S/s sampling rate. Comparative results obtained for E-textile dry and normal ECG are presented in Fig. 6 and Fig. 7.

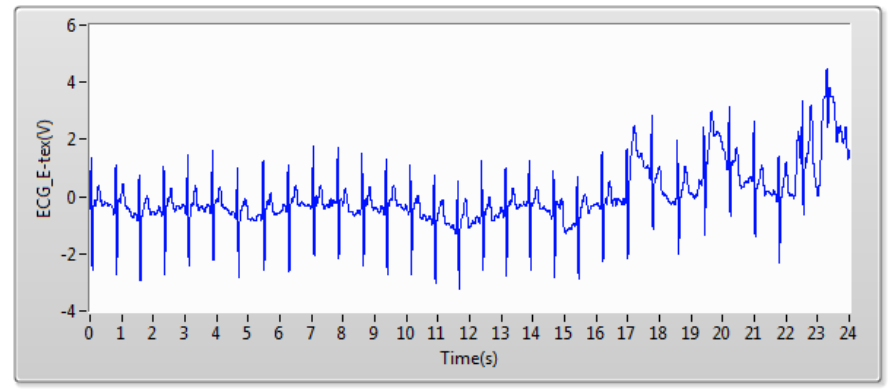

Figure 6. Signal acquired with E-textile dry electrodes and Medlab POX100 reference ECG measurement system for 24 s time interval.

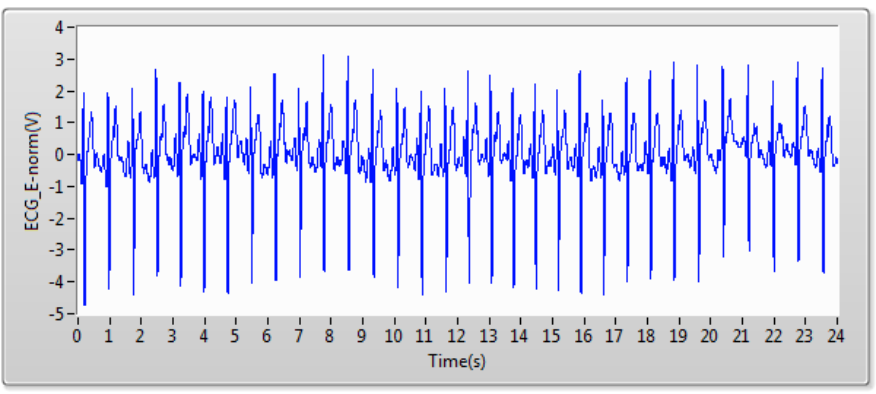

Figure 7. Signal acquired with normal ECG electrodes and Medlab P-OX100 reference ECG measurement system for $24 \mathrm{~s}$ time interval. 
As it can be observed in Fig. 6 and Fig. 7, the usage of Etextile dry electrodes conducts to smaller signal peak-to-peak values and bigger artefacts associated with respiratory activity and the with the T-shirt user motion. To improve the T-shirt capability special attention will be granted to the implementation of the T-shirt in terms of comfort and measurement reliability. A tight fit may be pleasant and wished by a professional or amateur athlete, but in the case of the elderly or disabled people it may be a factor of discomfort. A careful design of the shirt can provide ways of applying pressure to the critical points without compromising the overall comfort.

\section{SignAl Processing}

As can be observed in the previous figures (Fig. 4, 5, 6, 7), important artefacts are present in the acquired ECG signals when the E-textile dry electrodes or normal (wet) ECG electrodes are used, but also when a set of wet electrodes as reference electrodes are used. In order to extract accurate information regarding the cardiac activity such as heart rate (HR) and heart rate variability (HRV), digital filtering (IIR Butterworth band pass filter) were designed and implemented as part of LabVIEW software for ECG processing.

Considering the robustness of wavelets based methods, a WA Detrend from LabVIEW Advanced Signal Processing Toolkit was employed. The obtained results for E-textile dry electrodes when the Medlab P-OX100 was used to acquire the ECG wave are presented in Fig. 8 .

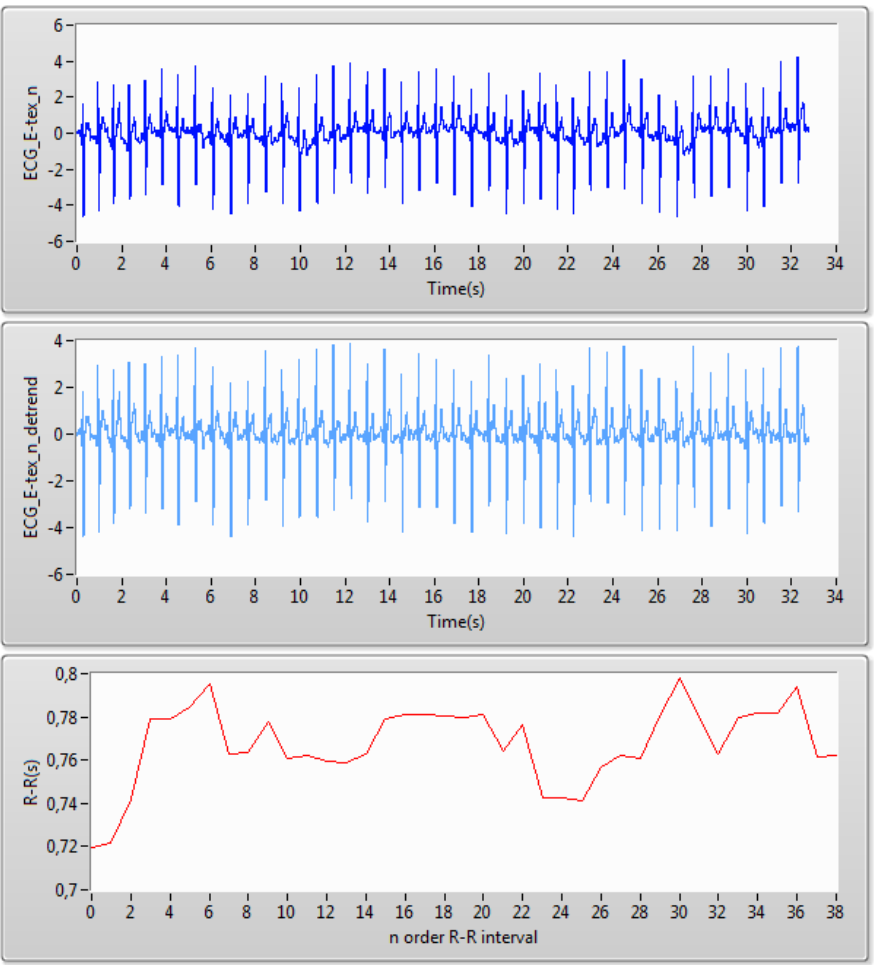

Figure 8. Dry electrodes ECG normalized signal (top), detrend signal (midle) and R-R interval variation (bottom).
Using a PeakDetector LabVIEW function and an adaptive threshold the R-R intervals associated with ECG QRST complex were calculated as the starting point for the HRV estimation using statistical time-domain methods. These may be divided into two classes, (a) those derived from direct measurements of the NN intervals or instantaneous heart rate, and (b) those derived from the differences between NN intervals [10]. These variables may be derived from analysis of the total electrocardiographic recording or may be calculated using smaller segments of the recording period. The $\mathrm{NN}$ are denominated normal-to-normal intervals and represents all of intervals between adjacent QRS complexes resulting from sinus node depolarization. In the present work the standard deviation of the R-R intervals was used. Thus, for the particular case of 300s ECG recorded time, the evolution of the standard deviation calculated for $15 \mathrm{~s}$ time segments of the whole period is presented in Fig. 9.

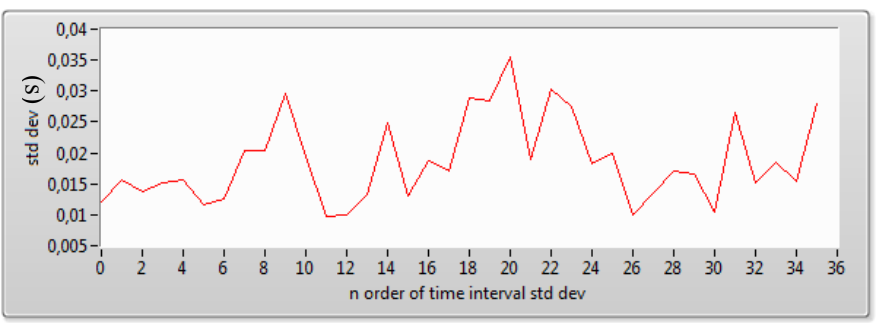

Figure 9. Standard deviation evolution as HRV estimator for analysed ECG wave corresponding to 300 s acquisition time.

The minimum, maximum and the mean of standard deviation calculated for R-R measured time interval were: $0.009 \mathrm{~s}$, $0.035 \mathrm{~s}$ and $0.019 \mathrm{~s}$. This statistics can give information about the HRV and can be easily implemented in embedded systems characterized by reduced processing capabilities.

\section{CONCLUSIONS}

The signals obtained using the developed shirt show acceptable quality even when compared to standard gel electrodes, although the difference between the dry and wet state of the sensing areas produces significant differences between the results. There are several factors to be considered in the design of the shirts according to the target user. A tight fit might be adequate and comfortable for the athlete and electrode positioning may be optimised for this case, but for the resting patient a looser fit and a different positioning of the electrodes may provide a good signal for unobtrusive health monitoring. It is important to stress that psychological factors are very important in this context. An elderly person may be averse to the application of electrodes, but wearing a "normal" shirt is a way of integrating healthcare in a gentler manner.

\section{REFERENCES}

[1] M. Catrysse, R. Puers, C. Hertleer, L. van Langenhove, H. van Egmond, D. Matthys, "Towards the integration of textile sensors in a wireless monitoring suit", Sensors and Actuators A 114, 302-311, 2004.

[2] M. J. Abreu, H. Carvalho, A. Catarino, A. Rocha, "Integration And Embedding of Vital Signs Sensors and Other Devices into Textiles", 
Medical and Healthcare Textiles, Woodhead Publishing Textile Series Nr.75, Editors J. F. Kennedy, S. C. Anand, M. Miraftab and S. Rajendran,, ISBN 184569224 1, July2010 .

[3] Silva, M., Catarino, A., Carvalho, H., Rocha, A., Monteiro, J., Montagna, G., "Textile Sensors for ECG and Respiratory Frequency on Swimsuits", Proceedings of the Conference on Intelligent Textiles and Mass Customisation, Casablanca, Morocco, 12-14th November 2009, ISBN 978-9954-8878-1-4.

[4] Xu, P. J., Zhang, H. and Tao, X. M., "Textile-structured electrodes for electrocardiogram", Textile Progress,40:4,183 - 213, 2008.

[5] Jens Mühlsteff, Olaf Such, "Dry electrodes for monitoring of vital signs in functional textiles", Proceedings of the 26th Annual International Conference of the IEEE EMBS, San Francisco, USA, 2004.

[6] Montagna, G., Catarino, A., Carvalho, H., Rocha, A., "Study and Optimization of Swimming Performance in Swimsuit Designed with
Seamless Technology", Autex 2009 World Textile Conference, Izmir, Turkey, 2009, May 26- 28th.

[7] Silva, M., Catarino, A., Carvalho, H., Rocha, A., Monteiro, J., Montagna, G., "Study Of Vital Sign Monitoring With Textile Sensor In Swimming Pool Environment". Proceedings of the IECON 2009, The 35th Annual Conference of The IEEE Industrial Electronics Society, 3-5 November 2009, Porto, Portugal.

[8] Pereira, T., Silva,P. Carvalho,H.,Carvalho, M., "Textile moisture sensor matrix for monitoring of disabled and bed-rest patients", Proceedings of the IEEE Eurocon 2011 Conference, Lisbon, Portugal, April 2011.

[9] Enrique Mario Spinelli, Ramon Pallàs-Areny, and Miguel Angel Mayosky. Ac-coupled front-end for biopotential measurements. IEEE Transactions on Biomedical Engineering, 50(3):391 - 195, March 2003.

[10] Task Force, "Heart Rate Variability - Guidelines", European Heart Journal (1996) 17, 354-381. 